\title{
Pricing of Non-redundant Derivatives in a Complete Market*
}

\author{
Abdelhamid BIZID $^{\dagger} \quad$ Elyès JOUINI ${ }^{\ddagger}$ \\ Pierre-François KOEHL ${ }^{\S}$
}

February 25, 1999

\begin{abstract}
We consider a complete financial market with primitive assets and derivatives on these primitive assets. Nevertheless, the derivative assets are non-redundant in the market, in the sense that the market is complete, only with their existence. In such a framework, we derive an equilibrium restriction on the admissible prices of derivative assets. The equilibrium condition imposes a well-ordering principle restricting the set of probability measures that qualify as candidate equivalent martingale measures. This restriction is preference free and applies whenever the utility functions belong to the general class of Von-Neumann Morgenstern functions. We provide numerical examples that show the applicability of the restriction for the computation of option prices.
\end{abstract}

\footnotetext{
${ }^{*}$ We are indebted to the editor Marti Subrahmanyam and two anonymous referees for very constructive comments. We have also benefitted from conversations with Nour Meddahi. We would like to thank seminar participants at Université de Montréal, Washington University, HEC Montréal, Ecole Polytechnique de Tunisie, Institut Henri Poincaré, Trento Mathematical Finance Conference(1997), Aspet (FIQUAM) Conference (1998) as well as the participants at the Quantitative Methods of Finance Conference, Australia, 1997.

†CERMSEM-Université de Paris 1 Panthéon-Sorbonne

${ }^{\ddagger}$ CREST, 15 Boulevard Gabriel Peri, 92245 malakoff Cedex, FRANCE, CERMSEMUniversité de Paris 1 Panthéon-Sorbonne and Center for Economic Policy Research. This author gratefully acknowledges the financial support of INQUIRE-Europe.

$\S$ ENSAE and CREST
} 


\section{Introduction}

It is well-known that, in a complete financial market, there exists a representative agent. His existence is convenient for the determination of the Arrow-Debreu prices at the equilibrium, since they can be linked to the gradient of his utility function taken at his optimal wealth (equal to the total wealth of the economy). Note that the total wealth does not depend on the prices of the purely financial assets (i.e. assets with a total supply equal to zero). Then, it is possible to find links between the Arrow-Debreu prices and the prices of the primitive assets.

More precisely, we assume in this paper that there exists a unique primitive asset interpreted as a stock. We show that, for a fixed state of the world, the Arrow-Debreu prices associated with the possible successor states are decreasing with the expected dividends of this asset. Furthermore, if at each date, the stock returns do not depend on its price, the Arrow-Debreu prices are then decreasing with the stock price. In fact, this result can be easily extended to the general case, where there exist more than one non-purely financial asset; in that case, the Arrow-Debreu prices are decreasing with the total wealth. From a practical point of view, this approach can be particularly useful for index options, the market index being taken as a proxy of the total wealth. Our result constrains the risk-neutral probabilities, and as shown numerically, the intervals of admissible prices for the purely financial assets are greatly reduced in comparison with the unconstrained case.

Some papers in the literature suggest constraints on risk-neutral probabilities - or equivalently, on state-price deflators. For instance, Cochrane and Saà-Requejo (1997) impose upper bounds on the volatility of the state-price deflators. They motivate their restrictions by assuming that any trader will accept any portfolio with a Sharpe Ratio greater than a given constant (for instance, 1.0). In order to obtain the target value on the Sharpe Ratio, they argue that the common value on the markets of the Sharpe Ratio is equal to 0.5. This approach restricts the attitude of the agents towards risk . Even if this method can be easily implemented - as Sharpe Ratios are observable on the markets - the restrictions seem to be quite arbitrary from a theoretical point of view. In the following, we do not restrict the risk-aversion of the agents, since we deal with very general Von Neumann-Morgenstern utility functions. Aït-Sahalia and Lo (1998) propose a nonparametric estimation of the state-price deflators. Using the market information, they estimate an option-pricing formula, then differentiate twice this estimator with respect to the strike of the option. Under suitable regularity conditions, this last quantity converges to a state-price deflator.

Another direction in the literature investigates the hedging problem and, 
since perfect hedging is no more possible in incomplete markets, tries to find the nearest (in some sense) payoff to the considered one among all the possible strategies. This approach is investigated for different criteria by, among others, Bertsimas, Kogan and Lo (1997) and Schweizer (1992, 1995). Nevertheless, when there are frictions on the markets or when the markets are incomplete, it is well known that the hedging problem and the optimal consumption-investment problem can no more be separated. In this paper, we work directly on equilibrium allocations and on equilibrium prices. We do not separate the two problems and we do not specify an analytical form for the risk criterium. We only need to assume that each agent maximizes the expected value of his utility function, the specification of this function is useless.

Our approach is not similar to that of Black and Scholes (1973) where the market is complete without the redundant derivative assets. Indeed, we assume the market is complete only in the presence of these derivative assets. Our approach differs also from the fictitious completion proposed by Karatzas, Lehoczky, Shreve and Xu (1991). Indeed, our result does not depend on a particular choice for the utility functions of the agents. In our approach, the dividend process of the primitive asset is given exogenously. Then our results can be seen as the derivatives prices compatible with partial equilibrium conditions.

Pricing models have two important applications: the pricing of newly introduced assets and the arbitrage from mispricing of the existing assets. Our approach assumes that the market is at the equilibrium. Hence, it is particularly relevant for the arbitrage or for the pricing of some existing assets with misinformation on the prices. Last, this approach permits also to give restrictions on the prices of newly introduced assets under a condition of non modification of the stock price. Conversely, if the new asset is introduced at a price which does not satisfy our restrictions then the equilibrium stock price will be automatically modified as in Detemple and Selden (1991).

The paper is organized as follows: in section 1, we show the interest of our methodology on a simple example. In section 2 , we present the general model. Section 3 is devoted to the proof of our main result. Section 4 investigates numerical examples in a quadrinomial framework. We compute the intervals of admissible prices, obtained with the constrained and classical unconstrained methods, for different options in various frameworks: index options and options with stochastic volatilities, European as well as American. The results are very encouraging. 


\section{A Simple Example}

Before introducing our model, let us consider the simplest example of incomplete markets and let us explain the main ideas of the paper on this example.

More precisely, consider a simple one-period model where the sample space and the probability are

$$
\begin{gathered}
\Omega=\left\{\omega_{1}, \omega_{2}, \omega_{3}\right\} \\
P=(1 / 3,1 / 3,1 / 3)
\end{gathered}
$$

First, there are two assets in the market:

a primitive asset whose prices are

$$
\begin{aligned}
& p(0)=25 \text { at date } 0 \\
& p\left(\omega_{1}\right)=20, p\left(\omega_{2}\right)=30 \text { or } p\left(\omega_{3}\right)=40 \text { at date } 1
\end{aligned}
$$

and a risk-free asset defined by an interest rate $r=\frac{1}{19}$.

It is straightforward that the feasible range of the Radon-Nykodym derivative of the martingale measures with respect to $P$ is

$$
\frac{1}{3}\left(Q\left(\omega_{1}\right), Q\left(\omega_{2}\right), Q\left(\omega_{3}\right)\right)=\frac{1}{3}\left(0.368+Q\left(\omega_{3}\right), 0.632-2 Q\left(\omega_{3}\right), Q\left(\omega_{3}\right)\right)
$$

where

$$
Q\left(\omega_{3}\right) \in[0,0.316]
$$

If there is one unit of primitive asset and no other endowment in the economy, the total wealth $W$ is then equal to $p$.

Let us now assume that there is also a non-redundant derivative asset in zero net supply. The market is then complete and there exists a representative agent in this economy with a utility function $u$.

At the equilibrium, the maximization program of this agent leads to the following first-order conditions:

$$
Q\left(\omega_{1}\right)=\frac{u^{\prime}(20)}{u^{\prime}(25)} ; Q\left(\omega_{2}\right)=\frac{u^{\prime}(30)}{u^{\prime}(25)} ; Q\left(\omega_{3}\right)=\frac{u^{\prime}(40)}{u^{\prime}(25)} .
$$

Since $u$ is strictly-concave, we must have

$$
Q\left(\omega_{1}\right)>Q\left(\omega_{2}\right)>Q\left(\omega_{3}\right) .
$$

After imposing these restrictions, we get the feasible range of the risk-neutral probability

$$
Q\left(\omega_{3}\right) \in[0.088,0.2107]
$$


which is quite smaller than the range in the unrestricted case. Furthermore, note that these smaller bounds have been obtained only under an assumption on the existence of the derivative asset but without knowing anything on its price process. Consequently, this approach permits to obtain bounds on the price of the derivative asset, which are better than the usual arbitrage-free bounds.

More precisely, if the derivative is a call option with a strike equal to 30 , we obtain $[0.836,2.001]$ as bounds on its price at $t=0$ instead of $[0,3.002]$ when using the classical no-arbitrage argument.

\section{The Model}

Taken as primitive are a complete probability space $(\Omega, \mathcal{F}, P)$ where $\Omega$ is a finite set of events and a time horizon $T$. Let $F=\left\{\mathcal{F}_{t}\right\}_{t=0, \ldots, T}$ be a filtration modelling the information structure. We denote by $E[$.$] the expectation$ under $P$ and by $E_{t}[$.$] the conditional expectation under P$ with respect to $\mathcal{F}_{t}$. A date $t$-node $\sigma_{t}$ is an atom of $\mathcal{F}_{t}$. We denote by $\Sigma_{t}$ the set of all date $t$-nodes and, for $\sigma_{t} \in \Sigma_{t}, \quad f\left(\sigma_{t}\right)=\left\{\sigma_{t+1} \in \Sigma_{t+1} ; \sigma_{t+1} \subset \sigma_{t}\right\}$ can be interpreted as the set of the immediate successors of the date $t$-node $\sigma_{t}$. For $t=0, \ldots, T-1$ and $\sigma_{t} \in \Sigma_{t}$, we define the transition probability of $P$ between dates $t$ and $t+1$ at the node $\sigma_{t}$ as follows

$$
\begin{aligned}
P_{\sigma_{t}}: & f\left(\sigma_{t}\right) \longrightarrow[0,1] \\
& \sigma_{t+1} \longmapsto P\left(\sigma_{t+1} \mid \sigma_{t}\right)
\end{aligned}
$$

We also define, for $t=0, \ldots, T-1$, the $\mathcal{F}_{t+1}$ measurable random variable $P_{t+1}$ by

$$
\forall \sigma_{t+1} \in \Sigma_{t+1}, P_{t+1}\left(\sigma_{t+1}\right)=P\left(\sigma_{t+1} \mid \sigma_{t}\right)
$$

where $\sigma_{t}$ is the unique element of $\Sigma_{t}$ such that $\sigma_{t+1} \in f\left(\sigma_{t}\right)$.

There is one nonstorable consumption good produced by one firm. At each date, the supply $d_{t}$ of this good is the production of the firm. This production is distributed as dividends to shareholders owning the firm. There is one equity claim which is tradable at date $t$, perfectly divisible and with an ex-dividend price $p_{t}$ in terms of consumption good. After the date $T$, the firm becomes obsolete and is valued at zero. A quantity $\theta$ of this claim insures to its owner a quantity $\theta d_{t}$ of the perishable good at date $t$. Throughout the paper, the total supply of the firm is normalized to one.

In addition to the equity claim described above, there are $m+1$ purely financial assets. By definition, their total supply is always zero. Moreover, for $i=0, \ldots, m$, the $i^{t h}$ purely financial asset is tradable at each date with a price $q_{t}^{i}$ in terms of consumption good. Those assets do not provide dividends. 
The processes $\left(p_{t}+d_{t}\right)_{t=0, \ldots, T}$ and $\left(\left(q_{t}^{i}\right)_{i=0, \ldots, m}\right)_{t=0, \ldots, T}$ are assumed to be positive and $F$-adapted. Furthermore, we assume that the $0^{\text {th }}$ purely financial asset is risk-free, i.e. the process $\left(R_{t}\right)_{t=1, \ldots, T}$ defined for all $t, t=0, \ldots, T-1$, by $\frac{1}{1+R_{t+1}}=\frac{q_{t}^{0}}{q_{t+1}^{0}}$ is anticipated.

There are $N$ consumers. The $n^{t h}$ consumer has an intertemporal utility function which associates the utility level $U^{n}($.$) with any F$-adapted consumption process $C=\left(C_{t}\right)_{t=0, \ldots T}$, as follows :

$$
U^{n}(C)=E\left[\sum_{t=0}^{T} u^{n}\left(C_{t}, t\right)\right]
$$

where $u^{n}$ maps $\mathbb{R}^{+*} \times\{0, \ldots, T\}$ in $\mathbb{R}$. We will require the following properties on $u^{n}$ :

Assumption 1 for all $n, u^{n}(., t)$ is a continuously differentiable increasing and strictly concave function. Moreover, we impose the following condition:

for $t=0, \ldots, T, \quad u^{n}(x ; t) \underset{x \rightarrow 0^{+}}{\rightarrow}-\infty$.

For any stopping time $\tau \leq T$, we define also the auxiliary utility level:

$$
U_{\tau}^{n}\left(\left(C_{t}\right)_{t=\tau, \ldots, T}\right)=E_{\tau}\left[\sum_{t=\tau}^{T} u^{n}\left(C_{t}, t\right)\right]
$$

A trading strategy $S$ is a vector

$$
\left\{\left(C_{t}\right)_{t=0, \ldots, T} ;\left(\theta_{t}\right)_{t=1, \ldots, T} ;\left(\alpha_{t}\right)_{t=1, \ldots, T}=\left(\left(\alpha_{t}^{i}\right)_{i=0, \ldots, m}\right)_{t=1, \ldots, T}\right\}
$$

where:

- $\forall t, t=0, \ldots, T, C_{t}$ is $\mathcal{F}_{t}$ measurable;

- $\forall t, t=1, \ldots, T, \theta_{t}$ and $\alpha_{t}$ are $\mathcal{F}_{t-1}$ measurable;

We interpret $\theta_{t}$ (resp. $\alpha_{t}^{i}$ ) as the quantity of the equity claim (resp. of the $i^{\text {th }}$ purely financial asset) owned at date $t$ and inherited from date $t-1$ by an agent following strategy $S$. In particular, $\alpha_{t}^{0}$ is the quantity of cash (including the interests) from date $t-1$ to date $t$, through the intermediary of the market interest rate. Furthermore, $\theta_{0}$ and $\alpha_{0}$ are the initial quantities of assets owned by the agent. We take, by convention, $\alpha_{T+1}^{i}=\theta_{T+1}=R_{T+1}=0$. 
The market schedule is: first, the firm produces and then distributes the dividends among the shareholders; second, consumption, new portfolios and new prices take place. Prices come from the equilibrium conditions, as defined in Assumption 2 below. We denote by $\alpha_{t} \cdot q_{t}$ the inner product between $\alpha_{t}$ and $q_{t}$. The budget constraint at dates $t=0, \ldots, T$ is:

$$
C_{t}+\theta_{t+1} p_{t}+\alpha_{t+1} \cdot q_{t}=\theta_{t}\left(p_{t}+d_{t}\right)+\alpha_{t} \cdot q_{t}=W_{t}
$$

where $W_{t}$ is interpreted as the wealth at date $t$ before consumption. It is a $\mathcal{F}_{t}$ measurable random variable.

An admissible strategy is a trading strategy $S$ satisfying the budget constraint (1) and the consumption constraint $C_{t} \geq 0$ for all $t$ between 0 and $T$. We denote by $\mathcal{A}$ the (convex) set of the admissible strategies.

Throughout the paper, we assume that there exists an equilibrium, i.e.:

Assumption 2 Given the utility functions and the initial endowments of the agents, there exists a vector:

$$
\left(S^{1}, \ldots, S^{N},\left(p_{t}+d_{t}\right)_{t=0, \ldots, T} ;\left(\left(q_{t}^{i}\right)_{i=0, \ldots, m}\right)_{t=0, \ldots, T}\right)
$$

such that $S^{n}$ is the strategy of agent $n$ and solves

$$
\sup _{S \in \mathcal{A}} U^{n}(C)
$$

where the budget constraints defining the set $\mathcal{A}$ are computed with the price processes $\left(p_{t}+d_{t}\right)_{t=0, \ldots, T}$ and $\left(\left(q_{t}^{i}\right)_{i=0, \ldots, m}\right)_{t=0, \ldots, T}$.

Furthermore, these strategies satisfy the market clearing conditions : $\sum_{1}^{N} S^{n}=\left\{\left(d_{t}\right)_{t=0, \ldots, T} ;(1)_{t=1, \ldots, T} ;(0)_{t=1, \ldots, T}\right\}$, i.e. at each date and in every state of the world, the total consumption is equal to the dividends, the total demand in the primitive asset is equal to 1 (the total supply) and the total demand in each purely financial asset is equal to zero.

We also assume that the financial market is complete. Formally:

Assumption 3 For any $F$-adapted process $x$, there exist $\left(\theta_{t}\right)_{t=0, \ldots, T}$ and $\left(\alpha_{t}\right)_{t=0, \ldots, T}$ such that:

- $\forall t, 1 \leq t \leq T, \theta_{t}$ and $\alpha_{t}$ are $\mathcal{F}_{t-1}$ measurable; $\theta_{0}$ and $\alpha_{0}$ are constant.

- $\forall t, 0 \leq t \leq T, \theta_{t}\left(p_{t}+d_{t}\right)+\alpha_{t} \cdot q_{t}-\theta_{t+1} p_{t}-\alpha_{t+1} \cdot q_{t}=x_{t}$, with the convention $\theta_{T+1}=\alpha_{T+1}=0$. 
Note that, in opposition to the Black and Scholes (1973) model, the financial assets may be non-redundant. We investigate the case where the price processes of the derivatives are not known because of a lack of information on the market. We prove in the rest of the paper that there exist constraints on the unobserved prices coming from the equilibrium analysis and independent of the agents' preferences.

\section{The Main Result}

In this section, we restrict the set of martingale probabilities used for the pricing of the financial assets. This result will be applied to specific underlying prices' dynamics and standard option prices in the next section.

We first give some useful definitions:

Definition 1 The discounted cum-dividends price processes of the underlying asset and of the $i^{\text {th }}$ financial asset are defined as follows:

$$
\begin{array}{r}
\left(\bar{p}_{t}\right)_{t=0, \ldots, T}=\left(p_{t} \prod_{j=1}^{t}\left(1+R_{j}\right)^{-1}+\sum_{j=0}^{t} d_{j} \prod_{k=1}^{j}\left(1+R_{k}\right)^{-1}\right)_{t=0, \ldots, T} \\
\left(\bar{q}_{t}^{i}\right)_{t=0, \ldots, T}=\left(q_{t}^{i} \prod_{j=1}^{t}\left(1+R_{j}\right)^{-1}\right)_{t=0, \ldots, T}
\end{array}
$$

Definition 2 We define the set $\mathcal{P}$ as the set of probability measures $Q$ equivalent to the true probability $P$ that transform the discounted process $\left(\bar{p}_{t}\right)_{t=0, \ldots, T}$ into a martingale.

Note that at the equilibrium there is no-arbitrage opportunities and the set $\mathcal{P}$ is then non-empty (see e.g. Duffie (1993), Theorem p.29).

Definition 3 We define the set $\mathcal{P}^{*}$ as the set of probability measures $Q \in \mathcal{P}$ such that:

$$
\begin{aligned}
\forall t=0, \ldots, T-1, \forall \sigma_{t} \in \Sigma_{t}, \forall\left(\sigma_{t+1}, \sigma_{t+1}^{\prime}\right) \in f\left(\sigma_{t}\right)^{2} \\
\quad\left(\frac{Q_{\sigma_{t}}\left(\sigma_{t+1}\right)}{P_{\sigma_{t}}\left(\sigma_{t+1}\right)}-\frac{Q_{\sigma_{t}}\left(\sigma_{t+1}^{\prime}\right)}{P_{\sigma_{t}}\left(\sigma_{t+1}^{\prime}\right)}\right)\left(d_{t+1}\left(\sigma_{t+1}\right)-d_{t+1}\left(\sigma_{t+1}^{\prime}\right)\right) \leq 0
\end{aligned}
$$

In other words, the set $\mathcal{P}^{*}$ is the set of probability measures in $\mathcal{P}$ such that, for every date $t$, the transition probabilities between a date $t$-node and its successors at date $t+1$ are in reverse order than the dividends at date $t+1$.

Our main result is: 
Theorem 4 Assume that there exists an equilibrium and that the financial market is complete. Then, under Assumption 1, we get: for any financial asset $i=1, \ldots, m$,

$$
q_{0}^{i} \in\left[\inf _{Q \in \mathcal{P}^{*}} E^{Q}\left[\bar{q}_{T}^{i}\right], \sup _{Q \in \mathcal{P}^{*}} E^{Q}\left[\bar{q}_{T}^{i}\right]\right]
$$

This theorem claims that we only have to consider a subset of the martingale measures set, namely the well-ordered ones, in order to price derivative assets. In the following, we will refer to this result as the "well-ordering principle". Intuitively, the underlying asset is in positive quantity in the economy, therefore it is held in a positive quantity by at least one risk-averse agent. Thus, the price of this asset has to be lower than its expected discounted value under the true probability, the difference being the risk-premium. It is easy to see that this is the case when the risk-neutral probability takes higher values when the underlying asset is cheaper. The converse is not obvious but holds as we shall see in the proof below.

Proof. Since the market is complete, there exists a representative agent (see e.g. Duffie (1993) Proposition p.26). More precisely, (Duffie (1993), Corollary 2 p.27), there exists $u$ satisfying Assumption 1 and mapping $\mathbb{R}^{+*} \times\{0, \ldots, T\}$ in $\mathbb{R}$, such that the utility level of the representative agent for an $F$-adapted consumption process $C=\left(C_{t}\right)_{t=0, \ldots, T}$ is

$$
U(C)=E\left[\sum_{t=0}^{T} u\left(C_{t}, t\right)\right]
$$

By definition of the representative agent, his optimal consumption process is equal, at each date, to the total consumption of the market, i.e. $C^{*}=$ $\left(d_{t}\right)_{t=0, \ldots, T}$, and his optimal portfolio is, at each date, the market portfolio, i.e. for all $t=0, \ldots, T, \theta_{t}^{*} \equiv 1$ and $\alpha_{t}^{*} \equiv 0$. We consider now the following auxiliary programs defined by backward induction:

$$
V_{T}(W)=u(W ; T)
$$

and, for all $t, t=0, \ldots, T-1$,

$$
\begin{array}{r}
V_{t}(W)=\max _{C_{t} \geq 0, \theta_{t+1}, \alpha_{t+1}} u\left(C_{t}, t\right)+E_{t}\left[V _ { t + 1 } \left(\left(W-C_{t}\right)\left(1+R_{t+1}\right)\right.\right. \\
+\theta_{t+1}\left(p_{t+1}+d_{t+1}-\left(1+R_{t+1}\right) p_{t}\right) \\
\left.\left.+\sum_{i=1}^{m} \alpha_{t+1}^{i}\left(q_{t+1}^{i}-\left(1+R_{t+1}\right) q_{t}^{i}\right)\right)\right]
\end{array}
$$

For all $t$ between 0 and $T, V_{t}$ maps $\mathbb{R}^{+}$in the space of the $\mathcal{F}_{t}$ measurable variables. In other words, $V_{t}(W)$ is the best the agent can do at date $t$ with 
the wealth $W$. We first prove that, for all $t, t=0, \ldots, T$, the function $V_{t}($.$) is$ increasing, concave and differentiable at the optimal value $W_{t}^{*}$. These results are established by backward induction.

Suppose that $V_{t+1}$ satisfies the properties. Immediately, $V_{t}$ is increasing and concave. Moreover, for all $W>0$,

$V_{t}(W) \geq u\left(C_{t}^{*}, t\right)+E_{t}\left[V_{t+1}\left(\left(W-C_{t}^{*}\right)\left(1+R_{t+1}\right)+p_{t+1}+d_{t+1}-\left(1+R_{t+1}\right) p_{t}\right)\right]$

and the equality holds for $W=W_{t}^{*}$. The function in the second term of the previous inequality is concave and differentiable with respect to $W$ at $W_{t}^{*}$ since $V_{t+1}$ is differentiable at the optimal value

$$
W_{t+1}^{*}=\left(W_{t}^{*}-C_{t}^{*}\right)\left(1+R_{t+1}\right)+\left(p_{t+1}+d_{t+1}-\left(1+R_{t+1}\right) p_{t}\right) .
$$

Therefore, according to Benveniste and Scheinkman (1979) (see also Stokey and Lucas (1989), p84), $V_{t}$ is differentiable at $W_{t}^{*}$ and:

$$
\begin{aligned}
V_{t}\left(W_{t}^{*}\right) & =u\left(C_{t}^{*}, t\right)+E_{t}\left[V_{t+1}\left(W_{t+1}^{*}\right)\right] \\
V_{t}^{\prime}\left(W_{t}^{*}\right) & =\left(1+R_{t+1}\right) E_{t}\left[V_{t+1}^{\prime}\left(W_{t+1}^{*}\right)\right]
\end{aligned}
$$

This ends the proof of the differentiability.

The optimal strategy at any date $t=0, \ldots, T$ for the representative agent achieves the maximum of the auxiliary program associated with $W=W_{t}^{*}=$ $\theta_{t}^{*}\left(p_{t}+d_{t}\right)+\alpha_{t}^{*} \cdot q_{t}$ since the budget constraints can be eliminated by replacing $\alpha_{t+1}^{0}$ as follows:

$$
\begin{aligned}
\alpha_{t+1}^{0} & =\frac{1}{q_{t}^{0}}\left[W_{t}-C_{t}-\theta_{t+1} p_{t}-\sum_{i=1}^{m} \alpha_{t+1}^{i} q_{t}^{i}\right] \\
& =\frac{1}{q_{t+1}^{0}}\left[W_{t+1}-\theta_{t+1}\left(p_{t+1}+d_{t+1}\right)-\sum_{i=1}^{m} \alpha_{t+1}^{i} q_{t+1}^{i}\right]
\end{aligned}
$$

For all date $t$, the differentiability of $V_{t}$ implies that we have (first order conditions of the maximization program with respect to $C_{t}, \theta_{t+1}$ and $\alpha_{t}$ ):

$$
\begin{gathered}
u^{\prime}\left(C_{t}^{*}, t\right)-\left(1+R_{t+1}\right) E_{t}\left[V_{t+1}^{\prime}\left(W_{t+1}^{*}\right)\right]=0 \\
E_{t}\left[\left(p_{t+1}+d_{t+1}-\left(1+R_{t+1}\right) p_{t}\right) V_{t+1}^{\prime}\left(W_{t+1}^{*}\right)\right]=0
\end{gathered}
$$

and for all $i=1, \ldots, m$,

$$
E_{t}\left[\left(q_{t+1}^{i}-\left(1+R_{t+1}\right) q_{t}^{i}\right) V_{t+1}^{\prime}\left(W_{t+1}^{*}\right)\right]=0
$$


For all date $t$, equations (4) and (5) lead to:

$$
u^{\prime}\left(C_{t}^{*}, t\right)=V_{t}^{\prime}\left(W_{t}^{*}\right)
$$

Therefore, the first order conditions can be rewritten

$$
p_{t}=\frac{1}{\left(1+R_{t+1}\right)} E_{t}^{Q}\left[\left(p_{t+1}+d_{t+1}\right)\right]
$$

and for all $i=1, \ldots, m$,

$$
q_{t}^{i}=\frac{1}{\left(1+R_{t+1}\right)} E_{t}^{Q}\left[q_{t+1}^{i}\right]
$$

where $E_{t}^{Q}[$.$] stands for the conditional expectation under a new probability$ $Q$ defined by the following density of the transition probabilities between dates $t$ and $t+1$ with respect to $P$ at node $\sigma_{t}$ :

$$
\begin{aligned}
Q_{\sigma_{t}}(.) & =\frac{V_{t+1}^{\prime}\left(W_{t+1}^{*}(.)\right)}{E_{t}\left[V_{t+1}^{\prime}\left(W_{t+1}^{*}\right)\right]} P_{\sigma_{t}}(.) \\
& =\frac{\left(1+R_{t+1}\right) u^{\prime}\left(C_{t+1}^{*}(.), t+1\right)}{u^{\prime}\left(C_{t}^{*}, t\right)} P_{\sigma_{t}}(.)
\end{aligned}
$$

Equations (6) and (7) prove that the discounted processes $\left(\bar{p}_{t}\right)_{t=0, \ldots, T}$ and $\left(\bar{q}_{t}^{i}\right)_{t=0, \ldots, T}$ are martingales under the probability $Q$. Moreover, given a node $\sigma_{t}$, the optimal wealth of the representative agent at any successor $\sigma_{t+1}$ of $\sigma_{t}$ is

$$
W_{t+1}^{*}\left(\sigma_{t+1}\right)=\left(p_{t+1}+d_{t+1}\right)\left(\sigma_{t+1}\right)
$$

and his optimal consumption is

$$
C_{t+1}^{*}\left(\sigma_{t+1}\right)=d_{t+1}\left(\sigma_{t+1}\right)
$$

Notice that the function $V_{t+1}$ in (8) depends on the node $\sigma_{t+1}$ (because of the backward induction), contrary to the function $u(., t+1)$. As $u(., t)$ is always concave, the density of probability is decreasing with the consumption according to (9). As $q_{t}^{0}$ is always strictly positive, we obtain that $Q \in \mathcal{P}^{*}$ and Result (2) immediately holds.

Therefore, if we do not know the price at date 0 of a given financial asset $q_{0}^{i}$, the interval of admissible prices is given by the infimum and the supremum over $\mathcal{P}^{*}$, instead of $\mathcal{P}$.

Before the computational results, we extend the Theorem to the cases where there are American derivatives, where there are more than one stock and where the agents have stochastic endowments. 


\section{Extensions}

\subsection{American derivatives.}

When American derivatives are allowed, we need to adapt Theorem 4, since the exercise time of an American derivative is not fixed anymore. Let us consider an exercise policy for the asset $i_{0}$ assumed to be American, associated to a stopping time $\tau$ bounded by $T$. Then, the payoff of the asset at the exercise is $q_{\tau}^{i_{0}}$. Adapting the proof of Theorem 4, it is easily checked that there exists $Q \in \mathcal{P}^{*}$ such that $q_{0}^{i_{0}}(\tau)=E^{Q}\left[\bar{q}_{\tau}^{i_{0}}\right]$ (the probability $Q$ is defined by the optimization program of the representative agent). An optimal exercise policy must be such that the associated stopping time achieves $\max _{\tau \leq T} q_{0}^{i_{0}}(\tau)$. Then, the following is proved:

Corollary 5 Assume that the $i_{0}^{\text {th }}$ asset is an American asset. Its price must lie in the interval:

$$
\left[\inf _{Q \in \mathcal{P}^{*}} \max _{\tau} E^{Q}\left[\bar{q}_{\tau}^{i_{0}}\right], \sup _{Q \in \mathcal{P}^{*}} \max _{\tau} E^{Q}\left[\bar{q}_{\tau}^{i_{0}}\right]\right]
$$

\subsection{Multiple risky assets}

Assume that there are $M(\geq 1)$ risky assets, with a total supply $\left(\xi^{j}\right)_{j=1, \ldots, M}$ in $\mathbb{R}_{+}$and a dividend process $\left(\left(d_{t}^{j}\right)_{j=1, \ldots, M}\right)_{t=0, \ldots, T}$ given exogenously. Assume also that there is a risk-free rate between dates $t$ and $t+1$ equal to $R_{t}$. As in section two, we want to constrain the price processes $\left(\left(q_{t}^{i}\right)_{i=1, \ldots, m}\right)_{t=0, \ldots, T}$ of $m$ purely financial assets. We define the market global dividend process by:

$$
\left(D_{t}\right)_{t=0, \ldots, T}=\left(\sum_{j=1}^{M} \xi^{j} d_{t}^{j}\right)_{t=0, \ldots, T}
$$

The analysis of the previous section, and in particular Theorem 4, amounts to change $\mathcal{P}$ and $\mathcal{P}^{*}$ as follows. We define $\mathcal{P}$ as the set of probability measures $Q$ equivalent to the true probability $P$ such that for any $j=1, \ldots, M$, the discounted process $\left(\bar{p}_{t}^{j}\right)_{t=0, \ldots, T}$ is martingale under the probability $Q$, and $\mathcal{P}^{*}$ as the set of probability measures $Q \in \mathcal{P}$ such that:

$\forall t=0, \ldots, T-1, \forall \sigma_{t} \in \Sigma_{t}, \forall\left(\sigma_{t+1}, \sigma_{t+1}^{\prime}\right) \in f\left(\sigma_{t}\right)^{2}$,

$$
\left(\frac{Q_{\sigma_{t}}\left(\sigma_{t+1}\right)}{P_{\sigma_{t}}\left(\sigma_{t+1}\right)}-\frac{Q_{\sigma_{t}}\left(\sigma_{t+1}^{\prime}\right)}{P_{\sigma_{t}}\left(\sigma_{t+1}^{\prime}\right)}\right)\left(D_{t+1}\left(\sigma_{t+1}\right)-D_{t+1}\left(\sigma_{t+1}^{\prime}\right)\right) \leq 0
$$


The restriction on the risk-neutral probabilities is then linked to the market global consumption only, even if we want to price a derivative on a single asset. This property comes from the use of the representative agent method. In fact, from a practical point of view, we can take the market index as a proxy of the global wealth.

More precisely, we have to consider the probability measures that make martingales all the price processes and satisfy the well-ordering principle with respect to the index dividends. When some price processes are not observable or when the index level is the only information available, we restrict our attention to the partial conditions given by the available information.

We provide a numerical example of an index option in the last section.

\subsection{Stochastic endowment}

The existence of stochastic endowments can be treated as well if the market global endowment process $\left(E_{t}\right)_{t=0, \ldots, T}$ is exogenously given. In that case, the analysis is exactly the same as in the previous subsection, including the market global endowment in the market global wealth and in the consumption of the representative agent.

In order to make this point clear, let us consider a simple example with two dates $(t=0, t=1)$ and three states of the world $\left(\omega_{1}, \omega_{2}, \omega_{3}\right)$. Suppose that the terminal payoff of the primitive asset is

$$
p\left(\omega_{1}\right)=20 ; p\left(\omega_{2}\right)=30 ; p\left(\omega_{3}\right)=40
$$

whereas its price at date 0 is equal to 1 . Assume that the risk-free rate is equal to 0 and that the terminal endowment is

$$
e\left(\omega_{1}\right)=0 ; e\left(\omega_{2}\right)=15 ; e\left(\omega_{3}\right)=0 .
$$

The total wealth of the economy (which is equal to the total consumption) is then

$$
W\left(\omega_{1}\right)=20 ; W\left(\omega_{2}\right)=45 ; W\left(\omega_{3}\right)=40
$$

and we have to take into consideration the probability measures $\left(\pi_{1}, \pi_{2}, \pi_{3}\right)$ such that

$$
\pi_{1} p\left(\omega_{1}\right)+\pi_{2} p\left(\omega_{2}\right)+\pi_{3} p\left(\omega_{3}\right)=1
$$

and

$$
\pi_{1} \geq \pi_{3} \geq \pi_{2}
$$

instead of

$$
\pi_{1} \geq \pi_{2} \geq \pi_{3}
$$


In fact, the probability measure has to satisfy a well ordering principle with respect to some permutation of the primitive asset dividends. If the stochastic endowment is strongly correlated to the dividend process of the underlying asset, we can expect that the introduction of the stochastic endowment will be innocuous.

\section{Computational Results in a Quadrinomial Framework}

In this section, we provide numerical examples in a quadrinomial framework. It enables us to consider general models with options written on an index and options with stochastic volatilities. We consider a $n$ time-steps tree. It is assumed, in all this section, that the distribution of the underlying asset's returns at each date is independent of the node. This means that there exists an $F$-adapted stochastic process $\left(\rho_{k T / n}\right)_{k=0, \ldots, n}$ such that

$$
\begin{aligned}
\rho_{0} & =1 \\
\text { for } k & =1, \ldots, n, \forall \sigma \in \Sigma_{k T / n}, \rho_{k T / n}=\frac{p_{k T / n}+d_{k T / n}}{p_{(k-1) T / n}}
\end{aligned}
$$

we will assume that $\rho_{k T / n}$ takes its values in $\left\{u_{1}, u_{2}, u_{3}, u_{4}\right\}$ and that the stock price at a given date is an increasing function of the dividend. If we assume that the distribution of the asset prices is known exogenously, we can choose $\left(p_{k T / n}\right)_{k=0, \ldots, n}$ as the underlying process. We consider then at each node the transitions $p_{k T / n} \rightarrow\left\{p_{(k+1) T / n}+d_{(k+1) T / n}\right\}$. The period discount rate $R$ is assumed to be constant, and we denote by $r$ the instantaneous discount rate which satisfies:

$$
e^{r T / n}=1+R
$$

\subsection{The general framework}

We consider a date $k T / n$-node $\sigma$ with four successors $\sigma_{1}, \sigma_{2}, \sigma_{3}, \sigma_{4}$. The underlying asset evolves as follows:

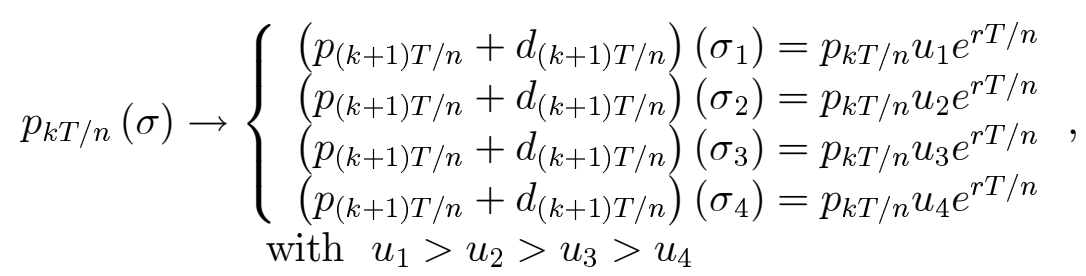


We will denote by $\left\{\pi_{i}\right\}_{i=1, \ldots, 4} \in(0,1)^{4}$ the true transition probabilities.

Let $\left\{\widehat{\pi}_{i}\right\}_{i=1, \ldots, 4} \in(0,1)^{4}$ be such that $\sum_{i=1}^{4} \widehat{\pi}_{i}=\sum_{i=1}^{4} \widehat{\pi}_{i} u_{i}=1$. The existence of such $\left\{\widehat{\pi}_{i}\right\}_{i=1, \ldots, 4}$ is guaranteed at the equilibrium by the classical no-arbitrage theorem and implies $u_{1}>1>u_{4}$. The two last equalities are equivalent to:

$$
\begin{aligned}
& \widehat{\pi}_{1}=\widehat{\pi}_{1} \\
& \widehat{\pi}_{2}=\widehat{\pi}_{2} \\
& \widehat{\pi}_{3}=\frac{\left(1-u_{4}\right)-\widehat{\pi}_{1}\left(u_{1}-u_{4}\right)-\widehat{\pi}_{2}\left(u_{2}-u_{4}\right)}{\left(u_{3}-u_{4}\right)} \\
& \widehat{\pi}_{4}=\frac{\left(u_{3}-1\right)+\widehat{\pi}_{1}\left(u_{1}-u_{3}\right)+\widehat{\pi}_{2}\left(u_{2}-u_{3}\right)}{\left(u_{3}-u_{4}\right)}
\end{aligned}
$$

Let $\widehat{\pi}_{1}$ and $\widehat{\pi}_{2}$ be given in $(0,1)$, since there exists at least one solution $\left\{\widehat{\pi}_{i}\right\}_{i=1, \ldots, 4}$, we only need to consider conditions $\widehat{\pi}_{3}>0$ and $\widehat{\pi}_{4}>0$. They are equivalent to:

$$
\widehat{\pi}_{2} \in\left[B_{\text {inf }}\left(\widehat{\pi}_{1}\right), B_{\text {sup }}\left(\widehat{\pi}_{1}\right)\right]
$$

where

$$
\begin{aligned}
& B_{\inf }\left(\widehat{\pi}_{1}\right)=\max \left(0, \frac{\left(1-u_{3}\right)-\widehat{\pi}_{1}\left(u_{1}-u_{3}\right)}{\left(u_{2}-u_{3}\right)}\right) \\
& B_{\text {sup }}\left(\widehat{\pi}_{1}\right)=\frac{\left(1-u_{4}\right)-\widehat{\pi}_{1}\left(u_{1}-u_{4}\right)}{\left(u_{2}-u_{4}\right)}
\end{aligned}
$$

For a given $\widehat{\pi}_{1} \in(0,1)$, one can find $\widehat{\pi}_{2}$ in $(0,1)$ if and only if:

$$
B_{\text {inf }}\left(\widehat{\pi}_{1}\right) \leq B_{\text {sup }}\left(\widehat{\pi}_{1}\right), B_{\text {inf }}\left(\widehat{\pi}_{1}\right) \leq 1 \text { and } 0 \leq B_{\text {sup }}\left(\widehat{\pi}_{1}\right)
$$

Finally, $\widehat{\pi}_{1}$ must be in the following interval

$$
\left[\max \left(0, \frac{1-u_{2}}{u_{1}-u_{2}}\right), \frac{1-u_{4}}{u_{1}-u_{4}}\right]
$$

Since we have a linear maximization program, extreme prices of the option with respect to $\left\{\widehat{\pi}_{i}\right\}_{i=1, \ldots, 4}$ will be achieved in one of the following points: If $u_{2}<1$,

$$
\begin{array}{lll}
\widehat{\pi}_{1}=\frac{1-u_{4}}{u_{1}-u_{4}} & \widehat{\pi}_{1}=\frac{1-u_{2}}{u_{1}-u_{2}} & \widehat{\pi}_{1}=\frac{1-u_{3}}{u_{1}-u_{3}} \\
\widehat{\pi}_{2}=0 & \widehat{\pi}_{2}=\frac{u_{1}-1}{u_{1}-u_{2}} & \widehat{\pi}_{2}=0 \\
\widehat{\pi}_{3}=0 & \widehat{\pi}_{3}=0 & \widehat{\pi}_{3}=\frac{u_{1}-1}{u_{1}-u_{3}} \\
\widehat{\pi}_{4}=\frac{u_{1}-1}{u_{1}-u_{4}} & \widehat{\pi}_{4}=0 & \widehat{\pi}_{4}=0
\end{array}
$$

If $u_{2}>1>u_{3}$

$$
\begin{array}{llll}
\widehat{\pi}_{1}=\frac{1-u_{4}}{u_{1}-u_{4}} & \widehat{\pi}_{1}=0 & \widehat{\pi}_{1}=0 & \widehat{\pi}_{1}=\frac{1-u_{3}}{u_{1}-u_{3}} \\
\widehat{\pi}_{2}=0 & \widehat{\pi}_{2}=\frac{1-u_{3}}{u_{2}-u_{3}} & \widehat{\pi}_{2}=\frac{1-u_{4}}{u_{2}-u_{4}} & \widehat{\pi}_{2}=0 \\
\widehat{\pi}_{3}=0 & \widehat{\pi}_{3}=\frac{u_{2}-1}{u_{2}-u_{3}} & \widehat{\pi}_{3}=0 & \widehat{\pi}_{3}=\frac{u_{1}-1}{u_{1}-u_{3}} \\
\widehat{\pi}_{4}=\frac{u_{1}-1}{u_{1}-u_{4}} & \widehat{\pi}_{4}=0 & \widehat{\pi}_{4}=\frac{u_{2}-1}{u_{2}-u_{4}} & \widehat{\pi}_{4}=0
\end{array}
$$


If $u_{3}>1$

$$
\begin{array}{lll}
\widehat{\pi}_{1}=\frac{1-u_{4}}{u_{1}-u_{4}} & \widehat{\pi}_{1}=0 & \widehat{\pi}_{1}=0 \\
\widehat{\pi}_{2}=0 & \widehat{\pi}_{2}=0 & \widehat{\pi}_{2}=\frac{1-u_{4}}{u_{2}-u_{4}} \\
\widehat{\pi}_{3}=0 & \widehat{\pi}_{3}=\frac{1-u_{4}}{u_{3}-u_{4}} & \widehat{\pi}_{3}=0 \\
\widehat{\pi}_{4}=\frac{u_{1}-1}{u_{1}-u_{4}} & \widehat{\pi}_{4}=\frac{u_{3}-1}{u_{3}-u_{4}} & \widehat{\pi}_{4}=\frac{u_{2}-1}{u_{2}-u_{4}}
\end{array}
$$

Now from Theorem 4, we can add the following requirement:

$$
\frac{\widehat{\pi}_{1}}{\pi_{1}} \leq \frac{\widehat{\pi}_{2}}{\pi_{2}} \leq \frac{\widehat{\pi}_{3}}{\pi_{3}} \leq \frac{\widehat{\pi}_{4}}{\pi_{4}}
$$

Using the same approach, we obtain:

$$
\widehat{\pi}_{2} \in\left[B_{\mathrm{inf}}^{\prime}\left(\widehat{\pi}_{1}\right), B_{\mathrm{sup}}^{\prime}\left(\widehat{\pi}_{1}\right)\right]
$$

where

$$
\begin{aligned}
& B_{\text {inf }}^{\prime}\left(\widehat{\pi}_{1}\right)=\max \left(\frac{\pi_{2}}{\pi_{1}} \widehat{\pi}_{1}, \frac{\left(1-u_{3}\right)-\widehat{\pi}_{1}\left(u_{1}-u_{3}\right)}{\left(u_{2}-u_{3}\right)}, \frac{\left(1-\pi_{1}-\pi_{2}\right)\left(1-u_{4}-\widehat{\pi}_{1}\left(u_{1}-u_{4}\right)\right)-\pi_{3}\left(u_{3}-u_{4}\right)\left(1-\widehat{\pi}_{1}\right)}{\left(1-\pi_{1}-\pi_{2}\right)\left(u_{2}-u_{4}\right)-\pi_{3}\left(u_{3}-u_{4}\right)}\right) \\
& B_{\text {sup }}^{\prime}\left(\widehat{\pi}_{1}\right)=\frac{\pi_{2}\left(\left(1-u_{4}\right)-\widehat{\pi}_{1}\left(u_{1}-u_{4}\right)\right)}{\pi_{2}\left(u_{2}-u_{4}\right)+\pi_{3}\left(u_{3}-u_{4}\right)}
\end{aligned}
$$

Finally:

$$
\widehat{\pi}_{1} \in\left[A_{\text {inf }}, A_{\text {sup }}\right]
$$

where

$$
\begin{aligned}
& A_{\text {inf }}=\max \left(0, \frac{\left(1-u_{2}\right)}{\left(u_{1}-u_{3}\right)}, \frac{\left(1-\pi_{1}\right)\left(1-u_{4}\right)-\pi_{2}\left(u_{2}-u_{4}\right)-\pi_{3}\left(u_{3}-u_{4}\right)}{\left(1-\pi_{1}\right)\left(u_{1}-u_{4}\right)-\pi_{2}\left(u_{2}-u_{4}\right)-\pi_{3}\left(u_{3}-u_{4}\right)}\right) \\
& A_{\text {sup }}=\frac{\pi_{1}\left(1-u_{4}\right)}{\pi_{1}\left(u_{1}-u_{4}\right)+\pi_{2}\left(u_{2}-u_{4}\right)+\pi_{3}\left(u_{3}-u_{4}\right)}
\end{aligned}
$$

In order for $A_{\text {inf }}$ to be lower than $A_{\text {sup }}$, one must check the following condition:

$$
\left(1-u_{2}\right)\left(\pi_{1} u_{1}+\pi_{2} u_{2}+\pi_{3} u_{3}+\pi_{4} u_{4}-u_{4}\right) \leq\left(u_{1}-u_{3}\right)\left(1-u_{4}\right) \pi_{1}
$$

which involves the true transition probabilities and is restrictive only if $u_{2}<1$.

We now apply the previous results to the pricing of options with stochastic volatility and to the pricing of index options. The tree-structure will be reconnecting and, except in the Poisson volatility process case, stationary.

\subsection{Stochastic volatility}

\subsubsection{Binomial diffusion process for the volatility}

We consider the following discrete process on a quadrinomial tree of $n$ timesteps

$$
p \rightarrow\left\{p u_{1} e^{r \frac{T}{n}}, p u_{2} e^{r \frac{T}{n}}, p u_{2}^{-1} e^{r \frac{T}{n}}, p u_{1}^{-1} e^{r \frac{T}{n}}\right\}, \text { with }\left\{\begin{array}{l}
u_{1}=e^{\sigma_{\max } \sqrt{\frac{T}{n}}} \\
u_{2}=e^{\sigma_{\min } \sqrt{\frac{T}{n}}}
\end{array}\right.
$$


This process is a discretization of the following diffusion process for the risky asset in a continuous-time market model:

$$
d S_{t}=r S_{t} d t+\sigma_{t} S_{t} d W_{t}
$$

where, at each date $t, \sigma_{t}$ is known to be equal almost everywhere to $\sigma_{\min }$ or to $\sigma_{\text {max }}$. We assume that for $i=1, \ldots, 4, \pi_{i}=\frac{1}{4}$. We are in a position to apply the general results established in the previous subsection with $u_{3}=u_{2}^{-1}$ and $u_{4}=u_{1}^{-1}$. If we do not take into account the well-ordering principle, the interval of admissible prices for the European Call is $\left[\mathrm{CRR}\left(\sigma_{\min }\right), \mathrm{CRR}\left(\sigma_{\max }\right)\right]$ where $\operatorname{CRR}(\sigma)$ is the price of a European Call in a binomial framework with a volatility $\sigma$. This result is due to the convexity of the payoff of the option with respect to the price of the underlying asset as shown by Avellaneda, Levy and Paras (1995). The same result holds for European and American Puts. If we take into account the well-ordering property, the interval of admissible prices converges to $\operatorname{CRR}\left(\sqrt{\frac{\left(\sigma_{\min }^{2}+\sigma_{\max }^{2}\right)}{2}}\right)$ for $n \rightarrow+\infty$. Indeed, we have at each node a maximization program on the three following points

$$
\begin{array}{lll}
\widehat{\pi}_{1}=\frac{u_{2}\left(u_{1}-1\right)}{u_{1}^{2} u_{2}+u_{1} u_{2}^{2}+u_{1}-3 u_{2}} & \widehat{\pi}_{1}=\frac{2 u_{1} u_{2}-u_{1}-u_{2}}{2\left(u_{1}^{2} u_{2}+u_{1} u_{2}^{2}-u_{1}-u_{2}\right)} & \widehat{\pi}_{1}=1-3 \widehat{\pi}_{2} \\
\widehat{\pi}_{2}=\widehat{\pi}_{1} & \widehat{\pi}_{2}=\widehat{\pi}_{1} & \widehat{\pi}_{2}=\frac{u_{1} u_{2}\left(u_{1}\right.}{3 u_{1}^{2} u_{2}-u_{1} u_{2}^{2}} \widehat{\pi}_{3}=\widehat{\pi}_{2} \\
\widehat{\pi}_{3}=\widehat{\pi}_{1} & \widehat{\pi}_{3}=\frac{1}{2}-\widehat{\pi}_{1} & \widehat{\pi}_{4}=\widehat{\pi}_{2}
\end{array}
$$

We consider an underlying asset such that $p_{0}=100, \sigma_{\min }=10 \%, \sigma_{\max }=$ $15 \%$. We assume also that the interest rate is $r=5 \%$. We want to price, in this framework, different kinds of options. The characteristics of these options are:

- a European Call at the money (i.e. $K=100$ ) with $T=1$ year

- a European Binary Call at the money $\left(q_{T}=1\right.$ if $p_{T}+d_{T} \geq 100, q_{T}=0$ otherwise) with $T=1$ year

- a European Put at the money with $T=1$ year

- an American Put at the money with $T=1$ year

- a European Up \& Out Call at the money with $T=1$ year and an up and out barrier of $L=120$ (i.e. if $\max _{0 \leq t \leq T}\left(p_{t}+d_{t}\right) \geq L$, the option value is zero). 
Tables 1 and 2 present the intervals of admissible prices for different options, when varying the number of time-steps $n$.

Table 3 shows more specifically the convergence property for a European Call, with respect to $n$.

We prove that we have a great stability of the results for various intervals of volatility. We find also that the pricing intervals are very tiny (Table 4 ).

This last result is due in part to the fact that the considered true probability (for $i=1, \ldots, 4, \pi_{i}=\frac{1}{4}$ ) makes the stock price process quasi-martingale. It can be seen from Table 5 that the discounted price of the underlying asset is close to the expectation, that is, the risk premium is close to zero.

The results obtained with varying true probabilities (or varying risk premia) are not so good, but satisfying. Indeed, suppose now that we consider the following example : $\pi_{1}=\frac{1}{8}, \pi_{2}=\frac{1}{2}, \pi_{3}=\frac{1}{8}, \pi_{4}=\frac{1}{4}$. In this case, the risk premium may be higher. We obtain the three following points on which we compute the maximization program at each node of the tree :

$$
\begin{array}{lll}
\widehat{\pi}_{1}=\frac{u_{2}\left(u_{1}-1\right)}{u_{1}^{2} u_{2}+4 u_{1} u_{2}^{2}+u_{1}-6 u_{2}} & \widehat{\pi}_{1}=\frac{3 u_{1} u_{2}-u_{1}-2 u_{2}}{3 u_{1}^{2} u_{2}+12 u_{1} u_{2}^{2}-5 u_{1}-10 u_{2}} & \widehat{\pi}_{1}=1-2 \widehat{\pi}_{2} \\
\widehat{\pi}_{2}=4 \widehat{\pi}_{1} & \widehat{\pi}_{2}=4 \widehat{\pi}_{1} & \widehat{\pi}_{2}=\frac{7 u_{1} u_{2}\left(u_{1}-1\right)}{7 u_{1}^{2} u_{2}-4 u_{1} u_{2}^{2}-u_{1}-2 u_{2}} \\
\widehat{\pi}_{3}=\widehat{\pi}_{1} & \widehat{\pi}_{3}=\frac{1}{3}\left(1-5 \widehat{\pi}_{1}\right) & \widehat{\pi}_{3}=\frac{1}{2} \widehat{\pi}_{2} \\
\widehat{\pi}_{4}=1-6 \widehat{\pi}_{1} & \widehat{\pi}_{4}=\frac{2}{3}\left(1-5 \widehat{\pi}_{1}\right) & \widehat{\pi}_{4}=\frac{1}{2} \widehat{\pi}_{2}
\end{array}
$$

The computational results are given for the European Call, a 100 time-steps tree, and varying intervals of volatility in Table 6 .

Note that, as the true probability is not close to a log-normal diffusion process, the convergence to $C R R\left(\sqrt{\frac{\left(\sigma_{\min }^{2}+\sigma_{\max }^{2}\right)}{2}}\right)$ does not hold anymore.

We consider now varying true probabilities depending linearly on a parameter $\varepsilon \in \mathbb{R}$, as follows: $\pi_{1}=\frac{1}{4}, \pi_{2}=\frac{1}{4}+\varepsilon, \pi_{3}=\frac{1}{4}, \pi_{4}=\frac{1}{4}-\varepsilon$. In this case, we can easily draw the risk premium as a function of the parameter $\varepsilon$ (see Figure 1).

Note that $\varepsilon$ lies by definition in the following closed interval [ $-0.0032581,0.25]$. We obtain the three following points on which we compute the maximization program at each node of the tree:

$$
\begin{array}{lll}
\widehat{\pi}_{1}=\frac{u_{2}\left(u_{1}-1\right)}{u_{1}^{2} u_{2}+(1+4 \varepsilon) u_{1} u_{2}^{2}+u_{1}-(3+4 \varepsilon) u_{2}} & \widehat{\pi}_{1}=1-3 \widehat{\pi}_{3} \\
\widehat{\pi}_{2}=(1+4 \varepsilon) \widehat{\pi}_{1} & \widehat{\pi}_{2}=(1+4 \varepsilon) \widehat{\pi}_{3} \\
\widehat{\pi}_{3}=\widehat{\pi}_{1} & \widehat{\pi}_{3}=\frac{u_{1} u_{2}\left(u_{1}-1\right)}{3 u_{1}^{2} u_{2}-(1+4 \varepsilon) u_{1} u_{2}^{2}-u_{1}-(1-4 \varepsilon) u_{2}} \\
\widehat{\pi}_{4}=1-(3+4 \varepsilon) \widehat{\pi}_{1} & \widehat{\pi}_{4}=(1-4 \varepsilon) \widehat{\pi}_{3}
\end{array}
$$




$$
\begin{aligned}
& \widehat{\pi}_{1}=\frac{2(1-2 \varepsilon) u_{1} u_{2}-u_{1}-(1-4 \varepsilon) u_{2}}{2\left((1-2 \varepsilon) u_{1} u_{2}\left(u_{1}+(1+4 \varepsilon) u_{2}\right)-(1+2 \varepsilon)\left(u_{1}+(1-4 \varepsilon) u_{2}\right)\right)} \\
& \widehat{\pi}_{2}=(1+4 \varepsilon) \widehat{\pi}_{1} \\
& \widehat{\pi}_{3}=\frac{1}{2(1-2 \varepsilon)}\left(1-2(1+2 \varepsilon) \widehat{\pi}_{1}\right) \\
& \widehat{\pi}_{4}=\frac{(1+4 \varepsilon)}{2(1-2 \varepsilon)}\left(1-2(1+2 \varepsilon) \widehat{\pi}_{1}\right)
\end{aligned}
$$

Figure 2 represents the pricing bounds for the European Call (with $\sigma_{\min }=$ $10 \%, \sigma_{\max }=15 \%$ and a 100 time-steps tree) and varying $\varepsilon$. The bounds widen as $\varepsilon$ increases (i.e. as the true probability moves away from the martingale case).

\subsubsection{Poisson diffusion process for the volatility}

The framework is very close to the previous one, except that we assume now that the transition probabilities depend on the value of $\sigma$. More precisely, if the volatility at date $t$ is equal to $\sigma_{\max }$, we have:

$$
\pi_{1}=\frac{(1-\lambda T / n)}{2} ; \pi_{2}=\frac{\lambda T / n}{2} ; \pi_{3}=\frac{\lambda T / n}{2} ; \pi_{4}=\frac{(1-\lambda T / n)}{2}
$$

and if it is equal to $\sigma_{\min }$, we have:

$$
\pi_{1}^{\prime}=\frac{\lambda T / n}{2} ; \pi_{2}^{\prime}=\frac{(1-\lambda T / n)}{2} ; \pi_{3}^{\prime}=\frac{(1-\lambda T / n)}{2} ; \pi_{4}^{\prime}=\frac{\lambda T / n}{2}
$$

where $\lambda$ is a positive parameter. We can interpret $\lambda$ as the Poisson parameter of the volatility process. Then, we must have $\lambda T<n$.

The general interval of admissible prices is the same as before, since it does not depend on the true transition probabilities. For the determination of the well-ordered interval of admissible prices, there are two cases:

- If the volatility of the risky asset is $\sigma_{\max }$, the maximization program is on the three points

$$
\begin{array}{ll}
\widehat{\pi}_{1}=\frac{(1-\lambda / n)\left(u_{1}-1\right) u_{2}}{\left(u_{1}^{2}-1\right) u_{2}-\lambda / n\left(u_{1}-u_{2}\right)\left(u_{1} u_{2}-1\right)} & \widehat{\pi}_{1}=\frac{\left(u_{1}-1\right) u_{2}-\lambda / n\left(u_{1}-u_{2}+u_{1} u_{2}\left(u_{2}-1\right)\right)}{\left(u_{1}^{2}-1\right) u_{2}+\lambda / n\left(u_{1}-u_{2}\right)\left(u_{1} u_{2}-1\right)} \\
\widehat{\pi}_{2}=\frac{\lambda / n}{(1-\lambda / n)} \widehat{\pi}_{1} & \widehat{\pi}_{2}=\left(1-\widehat{\pi}_{1}\right) \frac{\lambda / n}{(1+\lambda / n)} \\
\widehat{\pi}_{3}=\frac{\lambda / n}{(1-\lambda / n)} \widehat{\pi}_{1} & \widehat{\pi}_{4}=\left(1-\widehat{\pi}_{1}\right) \frac{\lambda / n}{(1+\lambda / n)} \\
\widehat{\pi}_{4}=1-\frac{(1+\lambda / n)}{(1-\lambda / n)} \widehat{\pi}_{1} & \\
\widehat{\pi}_{1}=\frac{\left(\left(u_{1}-1\right) u_{2}-\lambda / n\left(u_{1}-u_{2}\right)\right)(1-\lambda / n)}{(1-\lambda / n)} \\
\widehat{\pi}_{2}=\frac{\lambda-1) u_{2}-\lambda / n\left(u_{1}-u_{2}\right)\left(u_{1} u_{2}+1\right)}{(1+\lambda / n)} \\
\widehat{\pi}_{3}=\lambda / n\left(1-\frac{1}{(1-\lambda / n)}\right. & \\
\widehat{\pi}_{4}=(1-\lambda / n)-\widehat{\pi}_{1} &
\end{array}
$$


- The other case is obtained symmetrically, by changing $\lambda / n$ into $1-\lambda / n$.

We compute the intervals of admissible prices for $n=100$ where the volatility is initially equal to $\sigma_{\min }=10 \%$ or $\sigma_{\max }=15 \%$ and the risk-free rate is $r=5 \%$. The options considered are a European Call at the money with characteristics $K=100, T=1$ year and European and American Puts at the money with characteristics $K=100, T=1$ year. In Figures 3,4 and 5 , we provide the intervals with a varying parameter $\lambda$.

Note that the model can be easily generalized using different jump probabilities $\lambda_{1}$ and $\lambda_{2}$ for the transitions $\sigma_{\max } \rightarrow \sigma_{\min }$ and $\sigma_{\min } \rightarrow \sigma_{\max }$. We can also study the more general case where $\lambda$ lies in the interval $\left[\lambda_{\min }, \lambda_{\max }\right]$. We consider now the pricing of an index option with a constant volatility.

\subsection{Option on Index}

We consider a market consisting in three basic assets: a risk-free asset and two risky assets non perfectly correlated. We want to price in this setting a call on the Index: $\left(p_{1}+d_{1}+p_{2}+d_{2}-K\right)^{+}$. In the discrete-time model, we suppose that each asset follows a binomial stationary tree, i.e. for $i=1,2$, $p_{i} \rightarrow\left\{p_{i} u_{i} e^{r T / n}, p_{i} u_{i}^{-1} e^{r T / n}\right\}$ where $u_{i}=\exp \left(\sigma_{i} \sqrt{\frac{T}{n}}\right)$ and $\sigma_{1}>\sigma_{2}$. We suppose also that $p_{1}(0)=p_{2}(0)=p$. We apply the general model where the index plays the rule of the primitive asset. Each node presents then four (distinct) transitions:

$$
p_{1}+p_{2} \rightarrow\left\{\begin{array}{l}
p_{1} u_{1} e^{r T / n}+p_{2} u_{2} e^{r T / n} \\
p_{1} u_{1} e^{r T / n}+p_{2} u_{2}^{-1} e^{r T / n} \\
p_{1} u_{1}^{-1} e^{r T / n}+p_{2} u_{2} e^{r T / n} \\
p_{1} u_{1}^{-1} e^{r T / n}+p_{2} u_{2}^{-1} e^{r T / n}
\end{array}\right.
$$

We denote by:

$$
\begin{aligned}
& \alpha_{1}=\frac{p_{1} u_{1}+p_{2} u_{2}}{p_{1}+p_{2}} ; \alpha_{2}=\max \left(\frac{p_{1} u_{1}+p_{2} u_{2}^{-1}}{p_{1}+p_{2}}, \frac{p_{1} u_{1}^{-1}+p_{2} u_{2}}{p_{1}+p_{2}}\right) \\
& \alpha_{3}=\min \left(\frac{p_{1} u_{1}+p_{2} u_{2}^{-1}}{p_{1}+p_{2}}, \frac{p_{1} u_{1}^{-1}+p_{2} u_{2}}{p_{1}+p_{2}}\right) ; \alpha_{4}=\frac{p_{1} u_{1}^{-1}+p_{2} u_{2}^{-1}}{p_{1}+p_{2}}
\end{aligned}
$$

We prove that $\alpha_{2}>1$ and $\alpha_{3}<1$. In order to find the general interval, we do the maximization program on the following points:

$$
\begin{array}{llll}
\widehat{\pi}_{1}=\frac{1-\alpha_{4}}{\alpha_{1}-\alpha_{4}} & \widehat{\pi}_{1}=0 & \widehat{\pi}_{1}=0 & \widehat{\pi}_{1}=\frac{1-\alpha_{3}}{\alpha_{1}-\alpha_{3}} \\
\widehat{\pi}_{2}=0 & \widehat{\pi}_{2}=\frac{1-\alpha_{3}}{\alpha_{2}-\alpha_{3}} & \widehat{\pi}_{2}=\frac{1-\alpha_{4}}{\alpha_{2}-\alpha_{4}} & \widehat{\pi}_{2}=0 \\
\widehat{\pi}_{3}=0 & \widehat{\pi}_{3}=\frac{\alpha_{2}-1}{\alpha_{2}-\alpha_{3}} & \widehat{\pi}_{3}=0 & \widehat{\pi}_{3}=\frac{\alpha_{1}-1}{\alpha_{1}-\alpha_{3}} \\
\widehat{\pi}_{4}=\frac{\alpha_{1}-1}{\alpha_{1}-\alpha_{4}} & \widehat{\pi}_{4}=0 & \widehat{\pi}_{4}=\frac{\alpha_{2}-1}{\alpha_{2}-\alpha_{4}} & \widehat{\pi}_{4}=0
\end{array}
$$


If we add the well-ordering condition and assume that the true transition probabilities are equal, we get that

$$
\begin{aligned}
\widehat{\pi}_{1} \in & {\left[\frac{p_{1}\left(1-u_{1}^{-1}\right)\left(2-u_{1}\right)+p_{2}\left(1-u_{2}^{-1}\right)\left(2-u_{2}\right)}{2\left(p_{1}\left(u_{1}-u_{1}^{-1}\right)+p_{2}\left(u_{2}-u_{2}^{-1}\right)\right)},\right.} \\
& \left.\frac{p_{1}\left(1-u_{1}^{-1}\right)+p_{2}\left(1-u_{2}^{-1}\right)}{2\left(p_{1}\left(u_{1}-u_{1}^{-1}\right)+p_{2}\left(u_{2}-u_{2}^{-1}\right)\right)}\right]
\end{aligned}
$$

We also have

$$
\widehat{\pi}_{2} \in\left[B_{\text {inf }}\left(\widehat{\pi}_{1}\right), B_{\text {sup }}\left(\widehat{\pi}_{1}\right)\right]
$$

where

$$
\begin{aligned}
& B_{\text {inf }}\left(\widehat{\pi}_{1}\right)=\max \left[\widehat{\pi}_{1}, \frac{2-\alpha_{3}-\alpha_{4}-\widehat{\pi}_{1}\left(2 \alpha_{1}-\alpha_{3}-\alpha_{4}\right)}{\left(2 \alpha_{2}-\alpha_{3}-\alpha_{4}\right)}\right] \\
& B_{\text {sup }}\left(\widehat{\pi}_{1}\right)=\frac{p_{1}\left(1-u_{1}^{-1}\right)+p_{2}\left(1-u_{2}^{-1}\right)}{\left(p_{1}\left(u_{1}-u_{1}^{-1}\right)+p_{2}\left(u_{2}-u_{2}^{-1}\right)\right)}-\widehat{\pi}_{1}
\end{aligned}
$$

We do then the following optimization program on three points if $\frac{2-\alpha_{3}-\alpha_{4}}{2\left(\alpha_{1}+\alpha_{2}-\alpha_{3}-\alpha_{4}\right)} \leq \frac{3-\alpha_{2}-\alpha_{3}-\alpha_{4}}{3 \alpha_{1}-\alpha_{2}-\alpha_{3}-\alpha_{4}}$

$$
\begin{array}{lll}
\widehat{\pi}_{1}=\frac{p_{1}\left(1-u_{1}^{-1}\right)\left(2-u_{1}\right)+p_{2}\left(1-u_{2}^{-1}\right)\left(2-u_{2}\right)}{2\left(p_{1}\left(u_{1}-u_{1}^{-1}\right)+p_{2}\left(u_{2}-u_{2}^{-1}\right)\right)} & \widehat{\pi}_{1}=\frac{p_{1}\left(1-u_{1}^{-1}\right)\left(2-u_{1}\right)+p_{2}\left(1-u_{2}^{-1}\right)\left(2-u_{2}\right)}{2\left(p_{1}\left(u_{1}-u_{1}^{-1}\right)+p_{2}\left(u_{2}-u_{2}^{-1}\right)\right)} \\
\widehat{\pi}_{2}=\widehat{\pi}_{1} & \widehat{\pi}_{2}=\frac{p_{1}\left(u_{1}-1\right)+p_{2}\left(u_{2}-1\right)}{2\left(p_{1}\left(u_{1}-u_{1}^{-1}\right)+p_{2}\left(u_{2}-u_{2}^{-1}\right)\right)} \\
\widehat{\pi}_{3}=\frac{\left(1-\alpha_{4}\right)-\widehat{\pi}_{1}\left(\alpha_{1}+\alpha_{2}-2 \alpha_{4}\right)}{\left(\alpha_{3}-\alpha_{4}\right)} & \widehat{\pi}_{3}=\frac{\left(1-\alpha_{4}\right)-\widehat{\pi}_{1}\left(\alpha_{1}-\alpha_{4}\right)-\widehat{\pi}_{2}\left(\alpha_{2}-\alpha_{4}\right)}{\left(\alpha_{3}-\alpha_{4}\right)} \\
\widehat{\pi}_{4}=\frac{\left(\alpha_{3}-1\right)+\widehat{\pi}_{1}\left(\alpha_{1}+\alpha_{2}-2 \alpha_{3}\right)}{\left(\alpha_{3}-\alpha_{4}\right)} & \widehat{\pi}_{4}=\frac{\left(\alpha_{3}-1\right)+\widehat{\pi}_{1}\left(\alpha_{1}-\alpha_{3}\right)+\widehat{\pi}_{2}\left(\alpha_{2}-\alpha_{3}\right)}{\left(\alpha_{3}-\alpha_{4}\right)} \\
\widehat{\pi}_{1}=\frac{p_{1}\left(1-u_{1}^{-1}\right)+p_{2}\left(1-u_{2}^{-1}\right)}{2\left(p_{1}\left(u_{1}-u_{1}^{-1}\right)+p_{2}\left(u_{2}-u_{2}^{-1}\right)\right)} & \\
\widehat{\pi}_{2}=\widehat{\pi}_{1} & \\
\widehat{\pi}_{3}=\widehat{\pi}_{1} & \\
\widehat{\pi}_{4}=1-3 \widehat{\pi}_{1} &
\end{array}
$$

and on four points otherwise

$$
\begin{array}{ll}
\widehat{\pi}_{1}=\frac{p_{1}\left(1-u_{1}^{-1}\right)\left(2-u_{1}\right)+p_{2}\left(1-u_{2}^{-1}\right)\left(2-u_{2}\right)}{2\left(p_{1}\left(u_{1}-u_{1}^{-1}\right)+p_{2}\left(u_{2}-u_{2}^{-1}\right)\right)} & \widehat{\pi}_{1}=\frac{p_{1}\left(1-u_{1}^{-1}\right)\left(2-u_{1}\right)+p_{2}\left(1-u_{2}^{-1}\right)\left(2-u_{2}\right)}{2\left(p_{1}\left(u_{1}-u_{1}^{-1}\right)+p_{2}\left(u_{2}-u_{2}^{-1}\right)\right)} \\
\widehat{\pi}_{2}=\frac{2-\alpha_{3}-\alpha_{4}-\widehat{\pi}_{1}\left(2 \alpha_{1}-\alpha_{3}-\alpha_{4}\right)}{\left(2 \alpha_{2}-\alpha_{3}-\alpha_{4}\right)} & \widehat{\pi}_{2}=\frac{p_{1}\left(u_{1}-1\right)+p_{2}\left(u_{2}-1\right)}{2\left(p_{1}\left(u_{1}-u_{1}^{-1}\right)+p_{2}\left(u_{2}-u_{2}^{-1}\right)\right)} \\
\widehat{\pi}_{3}=\frac{\left(1-\alpha_{4}\right)-\widehat{\pi}_{1}\left(\alpha_{1}-\alpha_{4}\right)-\widehat{\pi}_{2}\left(\alpha_{2}-\alpha_{4}\right)}{\left(\alpha_{3}-\alpha_{4}\right)} & \widehat{\pi}_{3}=\frac{\left(1-\alpha_{4}\right)-\widehat{\pi}_{1}\left(\alpha_{1}-\alpha_{4}\right)-\widehat{\pi}_{2}\left(\alpha_{2}-\alpha_{4}\right)}{\left(\alpha_{3}-\alpha_{4}\right)} \\
\widehat{\pi}_{4}=\frac{\left(\alpha_{3}-1\right)+\widehat{\pi}_{1}\left(\alpha_{1}-\alpha_{3}\right)+\widehat{\pi}_{2}\left(\alpha_{2}-\alpha_{3}\right)}{\left(\alpha_{3}-\alpha_{4}\right)} & \widehat{\pi}_{4}=\frac{\left(\alpha_{3}-1\right)+\widehat{\pi}_{1}\left(\alpha_{1}-\alpha_{3}\right)+\widehat{\pi}_{2}\left(\alpha_{2}-\alpha_{3}\right)}{\left(\alpha_{3}-\alpha_{4}\right)} \\
\widehat{\pi}_{1}=\frac{2-\alpha_{3}-\alpha_{4}}{2\left(\alpha_{1}+\alpha_{2}-\alpha_{3}-\alpha_{4}\right)} & \widehat{\pi}_{1}=\frac{p_{1}\left(1-u_{1}^{-1}\right)+p_{2}\left(1-u_{2}^{-1}\right)}{2\left(p_{1}\left(u_{1}-u_{1}^{-1}\right)+p_{2}\left(u_{2}-u_{2}^{-1}\right)\right)} \\
\widehat{\pi}_{2}=\widehat{\pi}_{1} & \widehat{\pi}_{2}=\widehat{\pi}_{1} \\
\widehat{\pi}_{3}=\frac{1}{2}-\widehat{\pi}_{1} & \widehat{\pi}_{3}=\widehat{\pi}_{1} \\
\widehat{\pi}_{4}=\frac{1}{2}-\widehat{\pi}_{1} & \widehat{\pi}_{4}=1-3 \widehat{\pi}_{1}
\end{array}
$$


We get the following results that we can compare with the two extremes (the general program and the risk-neutral world). Computationally, the bounds are given in Table 7, with $r=0 \%, \sigma_{1}=15 \%$ and $\sigma_{2}=10 \%$, for a European Call at the money, with a strike $K=200$

Table 8 provides results for a European and an American Put at the money with the same strike, but with a discount rate $r=5 \%$ (in order to change the optimal exercise policy) and 100 Steps

\section{Conclusion}

In this paper we have shown that the equilibrium conditions give strong restrictions on the admissible martingale-measures and then on the derivatives prices. The relevancy of these restrictions has been numerically exhibited on several examples dealing with different frameworks and derivative assets.

Note that the true probability is directly involved in our conditions, which is unusual. Nevertheless, some information has already been derived from the true probability since, as shown by Arrow (1970), and quoted by Huang and Litzenberger (1988, p.19), a risky asset can be chosen by an agent only if its expected rate of return (under the true probability measure) is greater than the risk-free asset return. It is then necessary to derive the true probability (identified with the historical one) from the data in order to apply our model.

We have not investigated the hedging of the derivative assets. When those assets are redundant, hedging comes to form a replicating portfolio. This is not the case in our model. The hedging strategy must be derived from the individual optimization problem. This requires to know the price process of every existing asset, which is obtained from the utility of the representative agent. This utility has to be estimated econometrically from the data.

At last we conjecture that any price lying in our constrained interval can be sustained at the equilibrium by a well-chosen utility function. We leave that point for further researches.

\section{References}

[1] Aït-Sahalia, Y. and A. W. Lo (1998), "Nonparametric estimation of state-price densities implicit in financial asset prices", Journal of Finance, 53, 499-547.

[2] Arrow, K. J. (1970), Essays in the Theory of Risk-Bearing, NorthHolland, Amsterdam. 
[3] Avellaneda, M., Levy, A. and A. Paras (1995), "Pricing and hedging derivative securities in markets with uncertain volatilities", Applied Mathematical Finance, 2, 73-88.

[4] Benveniste, L. M. and J. A. Scheinkman (1979), "On the differentiability of the value function in dynamic models of economics", Econometrica, 47, 727-732.

[5] Bertsimas, D., L. Kogan and A.W. Lo (1997), "Pricing and hedging derivative securities in incomplete markets : an $\varepsilon$-arbitrage approach", Working paper, Sloan School of Management, MIT.

[6] Bizid, A., E. Jouini and P.F. Koehl (1998), "Pricing in incomplete markets: an equilibrium approach", Working paper, CREST.

[7] Black, F. and M. Scholes (1973), "The pricing of options and corporate liabilities", Journal of Political Economy 81 637-654.

[8] Cochrane, J. H. and J. Saà-Requejo (1997), "Beyond arbitrage: "gooddeal" asset price bounds in incomplete markets", Working Paper.

[9] Detemple, J. and L. Selden (1991), "A general equilibrium analysis of option and stock market interactions", International Economic Review 32 279-303.

[10] Duffie, D. (1993), Dynamic Asset Pricing Theory, Princeton University Press, Princeton, New Jersey.

[11] Huang, C. F. and R. H. Litzenberger (1988), Foundations for Financial Economics, North-Holland, Amsterdam.

[12] Karatzas, I., Lehoczky, J. P., Shreve, S. E. and G.-L. Xu (1991), "Martingale and duality methods for utility maximization in an incomplete market", SIAM Journal of Control and Optimization, 29, 702-730.

[13] Schweizer, M., (1992), "Mean-variance hedging for general claims", Annals of Applied Probability, 2, 171-179.

[14] Schweizer, M., (1995), "Variance-optimal hedging in discrete time", Mathematics of Operations Research, 20, 1-31.

[15] Stokey, N. L. and R. E. Lucas (1989) Recursive Methods in Economic Dynamics, Harvard University Press, Cambridge, MA. 


\begin{tabular}{|l|l|l|l|l|l|}
\hline & Eur. Call & Bin. Call & Eur. Put & Am. Put & Up \& Out \\
\hline $\operatorname{CRR}\left(\sqrt{\frac{\sigma_{\min }^{2}+\sigma_{\max }^{2}}{2}}\right)$ & 7.764116 & 0.564304 & 2.887059 & 3.407614 & 3.001408 \\
\hline Well-ord. Lower Lim. & 7.770313 & 0.599351 & 2.893255 & 3.406393 & 2.970884 \\
\hline Well-ord. Upper Lim. & 7.779874 & 0.600587 & 2.902816 & 3.416010 & 2.987122 \\
\hline Arbitrage Lower Lim. & 6.812824 & 0.475717 & 1.935766 & 2.437191 & 1.702878 \\
\hline Arbitrage Upper Lim. & 8.602021 & 0.724580 & 3.724963 & 4.237087 & 4.899250 \\
\hline
\end{tabular}

Table 1 - Results for different kinds of options (with $n=100$ )

We consider here a quadrinomial reconnecting tree, which models a binomial structure of the volatility for the risky asset. In this Table, "CRR $\left(\sqrt{\frac{\sigma_{\min }^{2}+\sigma_{\max }^{2}}{2}}\right)$ ", is the asymptotic case, when the number of steps tends to infinity ; "Well-ord. Lower Lim." and "Well-ord. Upper Lim." are the bounds given by our approach at the equilibrium ; "Arbitrage Lower Lim." and "Arbitrage Upper Lim." are the bounds given by the arbitrage. Calculations are made for some representative options: an "at the money" (ATM) European Call ("Eur. Call"), an ATM Binary Call ("Bin. Call"), an ATM European Put ("Eur. Put"), an ATM American Put ("Am. Put") and an ATM Up \& Out Call ("Up \& Out"). Results are given for a tree of $n=500$ periods, with $\sigma_{\min }=10 \%$ and $\sigma_{\max }=15 \%$. The parameters values are a maturity $T=1$ year for each option and a discount factor $r=5 \%$. The initial price of the underlying asset is $p_{0}=100$. As the options are "at the money", their strike is equal to the initial price of the asset $k=100$. Moreover, the barrier of the Up \& Out Call is fixed at $L=120$. We get very tiny intervals with our approach and larger intervals considering only the arbitrage conditions. Note also that the asymptotic case is not always included in the interval, but that it is a good approximation. 


\begin{tabular}{|l|l|l|l|l|l|}
\hline & Eur. Call & Bin. Call & Eur. Put & Am. Put & Up \& Out \\
\hline $\operatorname{CRR}\left(\sqrt{\frac{\sigma_{\min }^{2}+\sigma_{\max }^{2}}{2}}\right)$ & 7.774589 & 0.601719 & 2.897531 & 3.412688 & 2.88974 \\
\hline Well-ord. Lower Lim. & 7.771180 & 0.598640 & 2.894122 & 3.410002 & 2.880769 \\
\hline Well-ord. Upper Lim. & 7.775451 & 0.599112 & 2.898394 & 3.414289 & 2.887712 \\
\hline Arbitrage Lower Lim. & 6.806426 & 0.495291 & 1.929368 & 2.436757 & 1.685774 \\
\hline Arbitrage Upper Lim. & 8.593191 & 0.708824 & 3.716134 & 4.233413 & 4.686262 \\
\hline
\end{tabular}

Table 2 - Results for different kinds of options (with $n=500$ )

We consider here a quadrinomial reconnecting tree, which models a binomial structure of the volatility for the risky asset. In this Table, "CRR $\left(\sqrt{\frac{\sigma_{\min }^{2}+\sigma_{\max }^{2}}{2}}\right)$ ", is the asymptotic case, when the number of steps tends to infinity ; "Well-ord. Lower Lim." and "Well-ord. Upper Lim." are the bounds given by our approach at the equilibrium ; "Arbitrage Lower Lim." and "Arbitrage Upper Lim." are the bounds given by the arbitrage. Calculations are made for some representative options: an "at the money" (ATM) European Call ("Eur. Call"), an ATM Binary Call ("Bin. Call"), an ATM European Put ("Eur. Put"), an ATM American Put ("Am. Put") and an ATM Up \& Out Call ("Up \& Out"). Results are given for a tree of $n=100$ periods, $\sigma_{\min }=10 \%$ and $\sigma_{\max }=15 \%$. The parameters values are a maturity $T=1$ year for each option and a discount factor $r=5 \%$. The initial price of the underlying asset is $p_{0}=100$. As the options are "at the money", their strike is equal to the initial price of the asset $k=100$. Moreover, the barrier of the Up \& Out Call is fixed at $L=120$. We get very tiny intervals with our approach. Note that, in comparison to Table 1, which gives results for a 100 steps tree, the equilibrium interval is more precise and the asymptotic case is more often included in the equilibrium interval. On the contrary, the arbitrage bounds do not seem to converge for a high number of steps. In fact, for convex pay-offs (standard European and American Calls or Puts), the lower bound converges to the Black and Scholes price associated to $\sigma_{\min }$ and the upper bound converges to the Black and Scholes price associated to $\sigma_{\max }$. For the other options (Binary Call and Up \& Out Call), one can only prove that the Black and Scholes bounds are included in the arbitrage interval. 


\begin{tabular}{|l|l|l|l|l|l|}
\hline & $n=10$ & $n=50$ & $n=100$ & $n=500$ & $n=1000$ \\
\hline $\operatorname{CRR}\left(\sqrt{\frac{\sigma_{\min }^{2}+\sigma_{\max }^{2}}{2}}\right)$ & 7.873366 & 7.791726 & 7.764116 & 7.774589 & 7.772933 \\
\hline Well-ord. Lower Lim. & 7.798492 & 7.771227 & 7.770313 & 7.771180 & 7.771484 \\
\hline Well-ord. Upper Lim. & 7.829619 & 7.784763 & 7.779874 & 7.775451 & 7.774504 \\
\hline
\end{tabular}

Table 3 - Convergence Results for the European Call

We compute a quadrinomial reconnecting tree, which models a binomial structure of the volatility for the risky asset. In this Table, "CRR $\left(\sqrt{\frac{\sigma_{\min }^{2}+\sigma_{\max }^{2}}{2}}\right)$ " is the asymptotic case, if the number of steps tends to infinity ; "Well-ord. Lower Lim." and "Well-ord. Upper Lim." are the bounds given by our equilibrium approach. We consider an "at the money" (ATM) European Call. Results are given for trees with various time-steps $n, \sigma_{\min }=10 \%$ and $\sigma_{\max }=15 \%$. The parameters values are adiscount factor $r=5 \%$ and a maturity $T=1$ year for the option. The initial price of the underlying asset is set at $p_{0}=100$ and the strike $k$ is also equal to 100 . We can see that the precision of the interval is increasing with the number of time-periods. 


\begin{tabular}{|l|l|l|}
\hline Interval $\left[\sigma_{\min }, \sigma_{\max }\right]$ & Well-Ordered Interval & Arbitrage Interval \\
\hline$[05 \%, 20 \%]$ & {$[8.426119,8.451166]$} & {$[5.279331,10.459286]$} \\
\hline$[06 \%, 19 \%]$ & {$[8.248757,8.270748]$} & {$[5.539258,10.088168]$} \\
\hline$[07 \%, 18 \%]$ & {$[8.094125,8.113088]$} & {$[5.833043,9.716874]$} \\
\hline$[08 \%, 17 \%]$ & {$[7.961263,7.977183]$} & {$[6.138075,9.345413]$} \\
\hline$[09 \%, 16 \%]$ & {$[7.852977,7.865789]$} & {$[6.469000,8.973792]$} \\
\hline$[10 \%, 15 \%]$ & {$[7.770313,7.779874]$} & {$[6.812824,8.602021]$} \\
\hline$[11 \%, 14 \%]$ & {$[7.715898,7.721955]$} & {$[7.156561,8.230107]$} \\
\hline$[12 \%, 13 \%]$ & {$[7.685186,7.687341]$} & {$[7.500204,7.858060]$} \\
\hline
\end{tabular}

Table 4 - Call Price Bounds for a varying range of $\left[\sigma_{\min }, \sigma_{\max }\right]$

We consider a quadrinomial reconnecting tree, which models a binomial structure of the volatility for the risky asset. In this Table, the "Well-ordered Interval" gives the bounds using our equilibrium approach and the "Arbitrage Interval" gives the bounds obtained only by the arbitrage conditions. We price an "at the money" (ATM) European Call ("Eur. Call"). Results are given for a tree of 100 periods. The parameters values are a discount factor $r=5 \%$ and a maturity $T=1$ year for the option. The initial price of the underlying asset is $p_{0}=100$ and the strike $k$ is also equal to 100 . We consider varying volatility values. We find that the arbitrage precision is very sensitive to the interval of volatilities (as it consists in the Black and Scholes prices for $\sigma_{\min }$ and $\left.\sigma_{\max }\right)$. It is not the case for the well-ordered interval, which gives very stable results. 


\begin{tabular}{|l|l|}
\hline Interval $\left[\sigma_{\min }, \sigma_{\max }\right]$ & Risk Premium \\
\hline$[05 \%, 20 \%]$ & $0.785549 \%$ \\
\hline$[06 \%, 19 \%]$ & $0.795628 \%$ \\
\hline$[07 \%, 18 \%]$ & $0.815789 \%$ \\
\hline$[08 \%, 17 \%]$ & $0.846038 \%$ \\
\hline$[09 \%, 16 \%]$ & $0.886385 \%$ \\
\hline$[10 \%, 15 \%]$ & $0.936840 \%$ \\
\hline$[11 \%, 14 \%]$ & $0.997420 \%$ \\
\hline$[12 \%, 13 \%]$ & $1.068141 \%$ \\
\hline
\end{tabular}

Table 5 - Risk premium of the stock

In Table 4, we compared the pricing intervals for different ranges of volatilities. We obtained very precise results even for wide ranges. This is in part due to our choice of true probability distribution. Indeed, for each node of the tree, the four possible states of the world at the following date are supposed equiprobable. This may a priori infer importantly on the computational results. A good way to measure the importance of the distribution of the true probability choice is to compute the risk premium of the risky asset. This risk premium is equal to the relative difference between the expected value of the asset at the final date and its initial price (more precisely: $\left.\left(E\left[p_{T}\right]-p_{0}\right) / p_{0}\right)$. We compute a quadrinomial tree of 100 steps as done in Table 4. One can see in this Table that the risk premium is very small in our model (almost one percent in a year). 


\begin{tabular}{|l|l|l|}
\hline Interval $\left[\sigma_{\min }, \sigma_{\max }\right]$ & Risk premium & Well-Ordered Interval \\
\hline$[05 \%, 20 \%]$ & $-5.280387 \%$ & {$[7.729859,7.907094]$} \\
\hline$[06 \%, 19 \%]$ & $-0.460844 \%$ & {$[7.706147,7.720148]$} \\
\hline$[07 \%, 18 \%]$ & $04.611808 \%$ & {$[7.569279,7.693189]$} \\
\hline$[08 \%, 17 \%]$ & $09.951244 \%$ & {$[7.457249,7.685950]$} \\
\hline$[09 \%, 16 \%]$ & $15.571895 \%$ & {$[7.392304,7.683990]$} \\
\hline$[10 \%, 15 \%]$ & $21.488996 \%$ & {$[7.382683,7.684766]$} \\
\hline$[11 \%, 14 \%]$ & $27.718625 \%$ & {$[7.441050,7.687538]$} \\
\hline$[12 \%, 13 \%]$ & $34.277757 \%$ & {$[7.578588,7.686510]$} \\
\hline
\end{tabular}

Table 6 - Call Price Bounds for a varying range of $\left[\sigma_{\min }, \sigma_{\max }\right]$

In order to see if the risk premium is important in the precision of our pricing method, we consider a different distribution of the true probability. In this Table, we compute simultaneously the risk premium and the pricing interval with our approach for different ranges of volatilities. We consider here a quadrinomial reconnecting tree, which models a binomial structure of the volatility for the risky asset. We price a standard European Call. Results are given for a tree of 100 periods. The parameters values are $r=5 \%$ and a maturity $T=1$ year for the option. The initial price of the underlying asset is $p_{0}=100$ and the strike $k$ is also equal to 100. We obtain also precise intervals for high risk premia. We consider also the case of negative risk premia (which is not a priori compatible with the arbitrage) because this case is realistic on short periods. The precision is also satisfying. 


\begin{tabular}{|l|c|c|}
\hline & 50 Steps & 100 Steps \\
\hline Risk_Neutral & 7.203377 & 7.201218 \\
\hline Well_Ordered & {$[7.118168,7.235547]$} & {$[7.134722,7.224525]$} \\
\hline General & {$[1.530502,9.950711]$} & {$[1.358856,9.965889]$} \\
\hline
\end{tabular}

Table 7 - European Call Prices on a Basket with different steps

We consider a quadrinomial reconnecting tree, which models an index on two risky assets. We assume that the first asset has the following characteristics: initial price $p_{0}^{1}=100$, volatility $\sigma_{1}=10 \%$. and that the second asset has an initial price $p_{0}^{2}=100$ and a volatility $\sigma_{2}=15 \%$. The correlation between the assets is not perfect and the exogenous information is only on the index diffusion process. We price a standard European Call on the index. Results are given for trees of 50 and 100 periods. The parameters values are $r=0 \%$ and a maturity $T=1$ year for the option. The initial price of the index is 200 and the strike $k$ is also equal to 200 (the Call is at the money). We give results in a risk neutral world. In this case, we know exactly the diffusion process of the two assets. The market is therefore complete and leads to a unique price for the option (row "Risk_Neutral"). Our method using the equilibrium is computed in row "Well_Ordered" and leads to a precise pricing interval. The precision is not satisfying if we use only the arbitrage conditions (row "General"). 


\begin{tabular}{|l|c|c|}
\hline & European Put & American Put \\
\hline Risk_Neutral & 3.176536 & 4.181391 \\
\hline Well_Ordered & {$[3.122208,3.196138]$} & {$[4.128643,4.201095]$} \\
\hline General & {$[0.000000,5.602376]$} & {$[0.354224,6.628533]$} \\
\hline
\end{tabular}

Table 8 - European and American Put Prices on a Basket

We consider in this Table a quadrinomial reconnecting tree, which models an index on two risky assets. We assume that the first asset has the following characteristics: initial price $p_{0}=100$, volatility $\sigma_{1}=10 \%$. and that the second asset has an initial price $p_{0}=100$ and a volatility $\sigma_{2}=15 \%$. The correlation between the assets is not perfect and the exogenous information consists only in the index diffusion process. We price a European Put and an American Put on the index. Results are given for a tree of 100 periods. The parameters values are $r=5 \%$ and a maturity $T=1$ year for the option. The initial price of the index is 200 and the strike $k$ in both cases is equal to 200 (the Puts are at the money). As the discount factor is not null, the prices of the European and the American options are different. We give results in a risk neutral world. In this case, we know the diffusion process of the two assets. The market is complete and we get a unique price for the option (row "Risk_Neutral"). Our method using the equilibrium is computed in row "Well_Ordered" and leads to a precise pricing interval. The precision is not satisfying if we use only the arbitrage conditions (row "General"). 
Figure 1:

Figure 2: 
Figure 3:

Figure 4: 
Figure 5: 